\title{
Preliminary Clinical Results of a Biphasic Waveform and an RV Lead System
}

\author{
ALI A. MEHDIRAD, CHARLES J. LOVE, MARSHALL S. STANTON, * \\ S. ADAM STRICKBERGER,† JAMES L. DUNCAN,‡ and MARK W. KROLL
}

From the Division of Cardiology, The Ohio State University, Columbus, Ohio; the *Mayo Clinic, Rochester, Minnesota; and the +University of Michigan Hospitals, Ann Arbor, Michigan; and the ‡Pacesetter Systems, Sylmar, California

MEHDIRAD, A.A., ET AL.: Preliminary Clinical Results of a Unique Biphasic Waveform and an RV Lead System. Biphasic defibrillation waveforms have provided a reduction in defibrillation thresholds in transvenous ICD systems. Although a variety of biphasic waveforms have been tested, the optimal pulse durations and tilts have yet to be identified. A multicenter clinical study was conducted to evaluate the performance of a new ICD biphasic waveform and new RV active fixation steroid eluting lead system. Fifty-three patients were entered into the study. Mean age was 63 years with a mean ejection fraction of $36.8 \%$. Primary indication for implantation was monomorphic ventricular tachycardia alone $(54.7 \%)$. Forty-eight patients (90.6\%) were implanted with an RV shocking lead and active can alone as the anodal contact. The ICD can was the cathode. In four cases (7.5\%), an additional SVC or CS lead was used due to a high DFT with the RV lead alone. In an additional case, a chronic SVC lead was used although the $R V$-Can DFT was acceptable. DFT for all cases at implant was $9.8 \pm 3.7 \mathrm{~J}$. Repeat testing at 3 months for a subset of patients showed a reduction in DFT $(7.4 \pm 3.0 \mathrm{~J}), P$ value $=0.03$. Sensing and pacing characteristics of the $R V$ lead system remained excellent during the study period (acute $0.047 \pm 0.005 \mathrm{~ms}$ at 5.4 $V$ and $9.9 \pm 6.2 \mathrm{mV} R$ wave; chronic $0.067 \pm 0.11 \mathrm{~ms}$ at $5.4 \mathrm{~V}$ and $9.3 \pm 5.4 \mathrm{mV} R$ wave). It is concluded that this lead system provides good acute and chronic sensing and pacing characteristics with good DFT values in combination with this waveform. (PACE 1999; 22[Pt.I]594-599).

implantable defibrillator, transvenous lead, steroid eluting lead, defibrillation threshold

\section{Introduction}

The use of implantable cardioverter defibrillator (ICD) technology in humans began with large abdominal implants using epicardial patches. ${ }^{1} \mathrm{Al}$ though the therapy was shown to be extremely effective, the requirement for a thoracotomy procedure to place the patch electrodes limited the clinical applicability. As the technology advanced, more advanced pectorally implanted devices with transvenous lead systems became available. ${ }^{2-5}$ Such advancements have now led to widespread clinical use of the ICD.

It is well accepted that some biphasic wave-

For the Aegis Investigators

Address for reprints: Ali A. Mehdirad, M.D., Mercy Cardiology, 621 South New Ballas Road, Suite 3005, St. Louis, MO, 63141. Fax: (314) 569-6594.

Received July 1, 1997; revised April 6, 1998; accepted June 12 , 1998. forms can decrease the defibrillation threshold (DFT) compared to monophasic waveforms. ${ }^{6.7}$ The shape and efficacy of biphasic waveforms can be altered by several factors including pulse amplitude, tilt, and durations. Studies in animal models and humans have demonstrated that some special biphasic waveforms can decrease DFT compared to other biphasic waveforms or a monophasic waveform, ${ }^{8-13}$ however the optimal biphasic waveform has yet to be identified in regard to pulse durations and tilt. This multicenter study evaluated a new biphasic waveform used with a new steroid eluting endocardial defibrillation lead (model 1559, Pacesetter Systems, Sylmar, CA, USA).

\section{Patients and Methods}

\section{Patient Population}

The study population included 53 successfully implanted patients. The mean age of the 
study population was $63.0 \pm 14.7$ years. There were 39 men in the group $(73.6 \%)$. Mean ejection fraction was $36.8 \% \pm 15.0 \%$ with a range of $13 \%-82 \%$. There were 14 patients $(26.4 \%)$ who were classified New York Heart Association (NYHA) Class I, 29 patients (54.7\%) were Class II, and 9 patients $(17.0 \%)$ were Class III. There were no patients identified as Class IV. In one case, data was not reported. Reported indications for implantation of the ICD included: monomorphic ventricular tachycardia (VT) alone (29 patients [54.7\%]); monomorphic VT and ventricular fibrillation (VF) (5 patients [9.4\%]); Polymorphic VT alone (3 patients [5.7\%]); polymorphic VT and VF (1 patient $[1.9 \%])$; and VF alone (15 [28.3\%]). The underlying cardiac disease was reported to be ischemic cardiomyopathy in 17 patients $(32.1 \%)$, dilated cardiomyopathy in 11 patients $(20.8 \%)$, combination of coronary artery disease and dilated cardiomyopathy in 9 patients $(17.0 \%)$, hypertrophic cardiomyopathy in 3 patients $(5.7 \%)$, valvular heart disease in 2 patients (3.8\%), other cardiomyopathies in 5 patients $(9.4 \%)$, and not reported in 6 patients $(11.3 \%)$. In 4 patients $(7.5 \%)$, an existing pacemaker system was in place. Fourteen patients $(26.4 \%)$ were on amiodarone and 6 patients $(11.3 \%)$ on sotalol. Twenty patients $(37.8 \%)$ were taking one cardioactive drug, and 16 patients $(30.2 \%)$ were taking two cardioactive drugs. There were 17 patients (32.1\%) on no cardioactive drugs.

\section{Waveform Description}

The biphasic waveform was incorporated in a new implantable ICD under clinical investigation (Aegis Model 2600, Pacesetter). The waveform is produced using $95 \mu \mathrm{F}$ capacitance (2 $190 \mu \mathrm{F}$ capacitors in series). Phase 1 of the waveform has a maximal leading edge voltage of $750 \mathrm{~V}$ with an initial tilt of $44 \%$ (Fig. 1). Following the $44 \%$ decay of the leading edge voltage, Phase 1 is extended another $1.6 \mathrm{~ms}$ before truncation. With typical shocking lead impedances, this provides a Phase 1 duration of approximately $4.5-5.0 \mathrm{~ms}$. The leading edge voltage of Phase 2 is equal to the trailing edge of Phase 1. The duration of Phase 2 is fixed at $2.5 \mathrm{~ms}$.

\section{Defibrillation Lead Description}

The Model 1559 lead system is a single endocardial screw-in lead which was placed in the

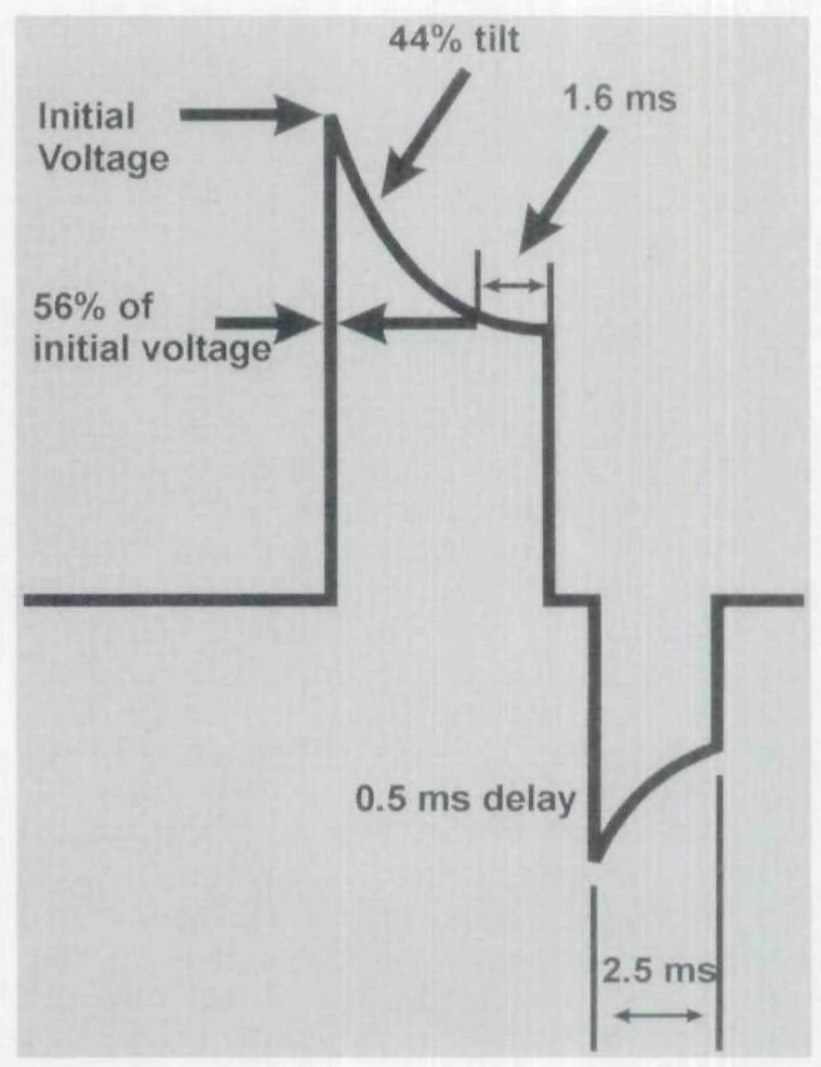

Figure 1. Biphasic waveform with $44 \%$ tilt followed by a fixed $1.6 \mathrm{~ms}$ extension in phase 1. Phase 2 is a fixed $2.5 \mathrm{~ms}$ pulse duration. Leading edge of phase 2 equals the trailing edge of phase 1.

right ventricular (RV) apex. The tip has an extendible/retractable screw that has a stimulating surface area of $8.5 \mathrm{~mm}^{2}$. Within the helix assembly is a silicone matrix plug that incorporates $1 \mathrm{mg}$ dexamethasone phosphate for gradual steroid elution. A ring electrode for true bipolar sensing capability is positioned $1 \mathrm{~cm}$ proximal from the tip and has a surface area of $34 \mathrm{~mm}^{2}$. Both the tip and ring electrodes are coated with a titanium nitride material to increase porosity, reduce polarization, and optimize sensing characteristics. The shocking coil is positioned $2.4 \mathrm{~cm}$ from the distal tip and is $5.0 \mathrm{~cm}$ in length. Total surface area of the shocking electrode is $459 \mathrm{~mm}^{2}$. The lead body is constructed of silicone rubber and has a diameter of $10 \mathrm{Fr}$. The two conductors for the tip and ring electrodes and the single shocking conductor are separately insulated within the lead body by silicone rubber. The rate sensing terminal is a stan- 
dard IS-1 connector and the high voltage connector is DF-1. Lead lengths are 58,85 , and $100 \mathrm{~cm}$.

The ICD physical dimensions were $8.1 \mathrm{~cm}$ wide, $6.3 \mathrm{~cm}$ high, and $1.6 \mathrm{~cm}$ thick providing a total volume of $60 \mathrm{cc}$. The weight of the device was $110 \mathrm{~g}$.

\section{Study Design}

The clinical trial included 14 participating centers in the U.S. and Europe. The study protocol required lead system electrical testing that included pacing thresholds and sensing amplitudes at implantation, predischarge from the hospital (typically the next day), and 1, 3, and 6 months postimplantation. Pacing and sensing values were determined both with external testing systems (PSA and DSA) and the ICD at the time of implantation. DFTs were measured at the time of device implantation using an external Defibrillation System Analyzer (DSA) and emulator can (Model 2670 DSA and model 2674 emulator, Pacesetter), that simulated the implanted device. The external system was used to allow control of the time from induction to shock for all patients. This provided better control for DFT measurements. The DFT was measured using a single step down protocol beginning at $600 \mathrm{~V}(17.1 \mathrm{~J})$ and stepping down by $100 \mathrm{~V}$ increments. When failure to defibrillate was observed, the next test shock was increased by 50 V. The DFT was then determined by the lowest energy test shock that resulted in successful conversion of the rhythm. If successful, conversion was observed at $4.2 \mathrm{~J}$, further testing to identify the DFT was not required. Repeat testing of DFT was repeated at the 3 month follow-up at the discretion of the investigator and patient. This data collection was not required and was therefore only collected on a subset of patients.

\section{Statistical Analysis}

A Student's paired $t$-test was used to compare acute and chronic DFT and sensing thresholds obtained in the "true" and "integrated" bipolar sensing configurations. A $\mathrm{P}$ value of $<0.05$ was considered significant.

\section{Results}

All 53 patients received the model 1559 RV defibrillation lead. The lead was typically im- planted on the left side (49 patients, $92.5 \%$ ) with the left subclavian vein predominately used ( 37 patients, $69.8 \%$ ). The lead was inserted via the cephalic vein in the remaining 12 patients $(22.6 \%)$ who had implants on the left side. Only three systems $(5.7 \%)$ were implanted on the right side (1 cephalic and 2 subclavian). In one case, the venous entry was not reported. Mean capture threshold at implant using an external pacing system analyzer (PSA) was $0.7 \pm 0.3 \mathrm{~V}(0.1-1.8)$ using a 0.5 $\mathrm{ms}$ pulse duration. Capture threshold was determined from the tip to the RV coil electrode as this was the configuration used by the implanted ICD for pacing. Mean pulse duration capture threshold using the DSA and implanted device was $0.065 \pm$ 0.09 and $0.047 \pm 0.005 \mathrm{~ms}$, respectively, at a fixed voltage of approximately $5.4 \mathrm{~V}$. As the device can be programmed to sense either "true" bipolar or "integrated" bipolar, both configurations were tested at implant. "True" bipolar uses both the tip and anodal band electrodes for sensing (tip-ring). "Integrated" bipolar uses the tip and defibrillation coil as the electrode pair (tip-coil). The mean R wave amplitude at implant for the tip-ring configuration ranged from 14.2-9.9 $\mathrm{mV}$ depending on the test method (PSA, DSA, or implanted ICD). Tip-coil configuration ranged from $11.6-4.6 \mathrm{mV}$. The tip-ring configuration provided statistically higher signals for all three test systems using the paired data (Table I).

In 48 patients (90.6\%), only the RV lead was implanted with the device. In these cases, the shocking configuration was RV coil (+) to device can (-). In 4 patients, a second transvenous lead was required due to an elevated DFT with the sin-

\section{Table I.}

Comparison of Implantation R Wave Measurements Between Tip-Ring ("True" Bipolar) and Tip-Coil ("Integrated" Bipolar) Configurations Using Paired Patient Data

\begin{tabular}{lccc}
\hline & PSA & DSA & Device \\
\hline Tip-Ring & $14.3 \pm 5.3$ & $10.3 \pm 4.8$ & $9.8 \pm 6.0$ \\
Tip-Coil & $11.7 \pm 5.5$ & $8.9 \pm 4.9$ & $4.7 \pm 2.9$ \\
P Value & 0.0001 & 0.0005 & 0.0001 \\
\hline
\end{tabular}

$\mathrm{DSA}=$ defibrillation system analyzer; $\mathrm{PSA}=$ pacing system analyzer 
gle RV lead to can configuration. In three of these cases a second shocking electrode was placed in the superior vena cava (SVC), and in one case, it was placed in the coronary sinus. In the final patient, there was an existing chronic SVC lead that was therefore utilized clinically. However, the RV to can configuration alone provided acceptable thresholds in this case.

The mean DFT at implant for all shocking configurations was $9.8 \pm 3.7 \mathrm{~J}(4.2-17.1)$. The DFT was $9.4 \pm 3.6 \mathrm{~J}$ for RV-can alone and $14.4 \pm 2.2 \mathrm{~J}$ for RVSVC (or CS)-can. The mean first shock impedance for the clinical settings was $53.5 \pm 10.8 \Omega$.

Sensing and capture thresholds on the defibrillation lead system remained stable during the chronic follow-up period. At the time of last follow-up, the $\mathrm{R}$ wave sensing amplitude (tip-ring) was $9.3 \pm 5.4 \mathrm{mV}(\mathrm{n}=42)$ as compared to the initial value through the implanted device of $9.9 \pm$ $6.2 \mathrm{mV}(\mathrm{n}=55)$. The $\mathrm{R}$ wave amplitude for the tipcoil configuration at last follow-up was $5.6 \pm 2.2$ $\mathrm{mV}(\mathrm{n}=3)$ as compared to $4.6 \pm 2.8 \mathrm{mV}(\mathrm{n}=54)$ at implant. Capture threshold at last follow-up using the implanted device was $0.067 \pm 0.11 \mathrm{~ms}$ at $5.4 \mathrm{~V}$ as compared to $0.047 \pm 0.005 \mathrm{~ms}$ at $5.4 \mathrm{~V}$ at implant.

Twelve patients had their DFT retested at the 3 month follow-up. Eleven of these patients showed the same or lower DFT at 3 months (Fig. 2). For the 12 patients, the mean implantation DFT was $9.8 \pm 3.0 \mathrm{~J}$ and the mean DFT at the 3 month

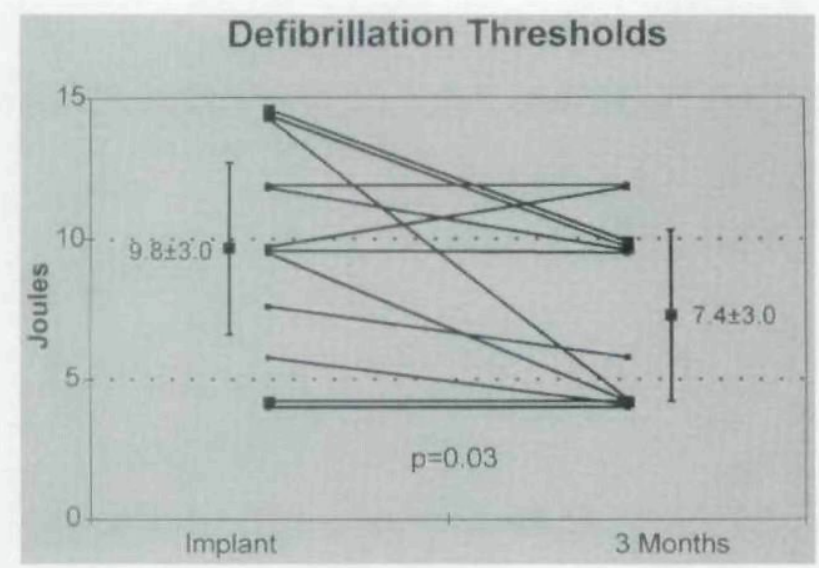

Figure 2. Paired defibrillation threshold data for 12 patients. There is a statistical reduction in threshold at 3 months. follow-up was $7.4 \pm 3.0 \mathrm{~J}$. These values were shown to be statistically different $(\mathrm{P}=0.03)$.

\section{Discussion}

Biphasic waveforms can lower DFTs compared to monophasic waveforms. Development of an optimal biphasic waveform should address many variables including capacitance, pulse durations, tilt, and amplitude of each phase.

The new waveform described differs from conventional biphasic waveforms in three areas: capacitance value, phase 1 duration, and phase 2 duration. The capacitor value is reduced to $95 \mu \mathrm{F}$ as compared to other ICD's using $125-180 \mu \mathrm{F}$. This change will increase the average current in phase 1 and give a duration of the primary phase close to the chronaxie, theoretically lowering the DFT. ${ }^{14-16}$ Several studies have demonstrated this relationship in animals ${ }^{17-18}$ and humans. ${ }^{19-23}$ The phase 1 duration is defined as a moderate tilt ( $44 \%)$ followed by a $1.6 \mathrm{~ms}$ extension. This provides a hybrid approach to the fixed tilt (which may overcompensate for impedance changes) and the fixed duration (which has no compensation). ${ }^{14}$ The phase 2 duration was fixed at $2.5 \mathrm{~ms}$ based on the "burping" theory that the function of phase 2 is to reduce the residual transmembrane potential from the phase 1 shock. ${ }^{24,25}$

In addition to sensing and pacing, the electrical performance of the ICD lead system can have an impact on the DFT. In our study, the electrical performance of the new lead system was found to be excellent during the 6 month study period. Sensing and capture thresholds remained clinically stable, possibly due to the steroid elution capability. However, a nonsteroid version of the lead was not prospectively compared.

Acute DFTs were found to be low and were seen to be significantly reduced at 3 months. Although the sample size in this study was small, the DFT results were favorable as compared to a larger reported study. ${ }^{26}$ In that study of 473 patients, the mean acute DFT was $11.4 \mathrm{~J}$ with a reported 3 month DFT of $9.9 \mathrm{~J}$. In this limited study, the acute DFT was $9.8 \mathrm{~J}$ and 3 month DFT was 7.4 $\mathrm{J}$. Due to the lack of randomization to other leads and waveforms in the study design, the level of contribution of the new waveform or new lead to the low DFT cannot be determined. 
Questions regarding the sensing performance of integrated bipolar systems have been raised before. ${ }^{27-28}$ In this limited study, the paired implantation data statistically revealed a reduction in the $\mathrm{R}$ wave amplitude for the integrated bipolar system. These measurements were made during normal sinus rhythm and not following a shock. Further degradation of the signal might have been seen postshock for the integrated bipolar system.

\section{Conclusion}

It is concluded that this new biphasic waveform provided a low acute and chronic DFT within this limited population. The sensing and pacing characteristics were also good and remained chronically stable. The steroid elution feature may have provided this benefit, but further prospectively randomized studies are needed.

\section{References}

1. Mirowski M, Reid PR, Mower MM, et al. Termination of malignant ventricular arrhythmias with an implanted automatic defibrillator in human beings. N Engl J Med 1980; 303:322-324.

2. Reddy RK, Bardy GH. Unipolar pectoral defibrillation systems. PACE 1997; 20:600-606.

3. Bardy GH, Hofer B, Johnson G, et al. Implantable transvenous cardioverter-defibrillators. Circulation 1993; 87:1152-1168.

4. Yee R, Klein GJ, Leitch JW, et al. A permanent transvenous lead system for an implantable pacemaker cardioverter-defibrillator: Nonthoracotomy approach to implantation. Circulation 1992; 85:196-204.

5. Trappe HJ, Klein H, Fieguth HG, et al. Initial experience with a new transvenous defibrillation system. PACE 1993; 16:134-140.

6. Kroll MW, Anderson KM, Supino CG, et al. Decline in defibrillation thresholds. PACE 1993; 16:213-217.

7. Saksena S, An H, Krol RB, et al. Simultaneous biphasic shocks enhance efficacy of endocardial cardioversion defibrillation in man. PACE 1991; 14:1935-1942.

8. Tang ASL, Yabe S, Wharton M, et al. Ventricular defibrillation using biphasic waveforms: The importance of phase duration. J Am Coll Cardiol 1989; 13:207-214.

9. Chapman PD, Vetter JW, Souza JJ, et al. Comparison of monophasic with single and dual capacitor biphasic waveforms for nonthoracotomy canine internal defibrillation. J Am Coll Cardiol 1989; 14:242-245.
Participating centers in this clinical trial included: The Ohio State University, Columbus, $\mathrm{OH}$ (A. Mehdirad, M.D.); The Mayo Clinic, Rochester, MN (M. Stanton, M.D.); University of Michigan Hospitals, Ann Arbor, MI (S.A. Strickberger, M.D.); Allegheny University, Philadelphia, PA (S. Kutalek, M.D.); Methodist Hospital, Lubbock, TX (G. Wells, M.D.); Sacred Heart Hospital, Eugene, OR (M. Antimisiaris, M.D.); St. Vincent's Hospital, Indianapolis, IN (E. Prystowsky, M.D.); University Hospital, Lucerne, Switzerland (R. Cardinas, M.D.); Na Homoloce, Prague, Czech Republic (M. Taborsky, M.D.); Sequoia Hospital, Redwood City, CA (H. Mead, M.D.); Luzern Hospital, Lucerne, Switzerland (P. Erne, M.D.); Michigan Capital Hospital, East Lansing, MI (R.K. Thakur, M.D.); St. Francis Hospital, Roslyn, NY (J. Levine, M.D.); and Huntington Memorial Hospital, Pasadena, CA (M. Meyers, M.D.).

10. Feeser SA, Tang ASL, Kavanaugh KM, et al. Strength-duration and probability of success curves for defibrillation with biphasic waveforms. Circulation 1990; 82:2128-2141.

11. Mehra R, DeGroot PJ, Norenbert MS. Energy waveforms and lead systems for implantable defibrillators. In B Luderitz, S Saksena (eds.): Interventional Electrophysiology. Armonk, NY, Futura Publishing Co., 1991, pp. 377-394.

12. Swartz JF, Fletcher RD, Karasik PE. Optimization of biphasic waveforms for human nonthoracotomy defibrillation. Circulation 1993; 88:2646-2654.

13. Natale A, Sra J, Krum D, et al. Relative efficacy of different tilts with biphasic defibrillation in humans. PACE 1996; 19:197-206.

14. Kroll MW. A minimal model of the monophasic defibrillation pulse. PACE 1993; 16:769-777.

15. Kroll MW, Lehmann MH, Tchou PJ. Defining the defibrillation dosage. In MW Kroll, MH Lehmann (eds.): Implantable Cardioverter Defibrillator Therapy: The Engineering-Clinical Interface. Dordrecht, Netherlands, Kluwer Academic Publishers, 1996, pp. 63-88.

16. Swerdlow CD, Brewer JE, Kass RM, et al. Estimation of optimal ICD capacitance from human strength-duration data. J Am Coll Cardiol 1997; 29:114A.

17. Leonelli FM, Kroll MW, Brewer JE. Defibrillation thresholds are lower with smaller storage capacitors. PACE 1995; 18:1661-1665.

18. Rist K, Tchou PJ, Mowrey K, et al. Smaller capacitors improve the biphasic waveform. J Cardiovasc Electrophysiol 1994; 5:771-776. 
19. Swerdlow CD, Kass RM, Hwang C, et al. Prospective randomized comparison of 60,90 , and $120 \mathrm{mi}-$ crofarad capacitors for unipolar defibrillation with retropectoral patch. Circulation 1994; 90:1229.

20. Swerdlow CD, Kass RM, Davie S, et al. Short biphasic pulses from 90 microfarad capacitors lower defibrillation threshold. PACE 1996; 19:1053-1060.

21. Swerdlow CD, Kass RM, Raissi S. Effect of capacitor size on defibrillation threshold in humans. J Am Coll Cardiol 1994; Special Issue:86A.

22. Swerdlow CD, Kass RM, Chen PS, et al. Effect of capacitor size and pathway resistance on defibrillation threshold for implantable defibrillators. Circulation 1994; 90:1840-1846.

23. Poole JE, Kudenchuk PJ, Dolack GL, et al. A prospective randomized comparison in humans of $90 \mu \mathrm{F}$ and $120 \mu \mathrm{F}$ biphasic pulse defibrillation using a unipolar defibrillation system. J Cardiovasc Electrophysiol 1995; 6:1097-1100.
24. Kroll MW. A minimal model of the single capacitor biphasic defibrillation waveform. PACE 1994; 17:1782-1792.

25. Swerdlow CD, Fan W, Brewer JE. Charge-burping theory correctly predicts optimal ratios of phase duration for biphasic defibrillation waveforms. Circulation 1996; 94:2278-2284.

26. Bardy GH, Yee R, Jung W, et al. Multicenter experience with a pectoral unipolar implantable cardioverter-defibrillator. J Am Coll Cardiol 1996; 28:400-410.

27. Peralta AO, John RM, Venditti FJ, et al. Undersensing of ventricular fibrillation in a noncommitted nonthoracotomy cardioverter defibrillator system. PACE 1997; 20:610-618.

28. Jung W, Manz M, Moosdorf R, et al. Failure of an implantable cardioverter-defibrillator to redetect ventricular fibrillation in patients with a nonthoracotomy lead system. Circulation 1992; $86: 1217-1222$. 
This document is a scanned copy of a printed document. No warranty is given about the accuracy of the copy. Users should refer to the original published version of the material. 